\section{Cambiando los paradigmas de la infección por M. pneumoniae en pediatría}

Merida-Vieyra $\mathrm{J}^{1,3}$, Aquino-Andrade $\mathrm{A}^{1}$, Ribas-Aparicio $\mathrm{RM}^{3}$, De ColsaRanero $\mathrm{A}^{1,2}$

\section{Resumen}

La neumonía atípica es un término que originalmente se utilizó en pacientes adultos que presentaban neumonías de curso atípico, generalmente leves y de evolución benigna, que eran causadas por agentes como: Mycoplasma pneumoniae, Chlamydophila pneumoniae y Legionella pneumophila.

En población pediátrica la presentación, tanto clínica como epidemiológica, de las neumonías por M. pneumoniae merece una especial atención ya que no siempre son cursos benignos y pueden existir numerosos cuadros extrapulmonares asociados; el proceso diagnóstico es complejo y poco diferencial de otros agentes etiológicos.

Recientemente ha llamado la atención la emergencia de la resistencia a macrólidos en algunos países del mundo, por lo que existen controversias respecto al tratamiento. Estudios numerosos realizados a nivel mundial, particularmente derivados de países asiáticos, han generado nueva información en relación al comportamiento biológico, clínico, epidemiológico, radiológico y respuesta terapéutica de $M$. pneumoniae en pacientes pediátricos.

PALABRAS CLAVE: Mycoplasma pneumoniae, pacientes pediátricos, neumonía, diagnóstico.

Acta Pediatr Mex. 2017 Nov;38(6):412-426.

\section{Changing the paradigms of M. pneumoniae infection in pediatrics.}

Merida-Vieyra $\mathrm{J}^{1,3}$, Aquino-Andrade $\mathrm{A}^{1}$, Ribas-Aparicio $\mathrm{RM}^{3}$, De ColsaRanero $\mathrm{A}^{1,2}$
Abstract
Atypical pneumonia is a term that was used originally in adult patients who had atypical pneumonia symptoms, usually with mild and benign courses, and were caused by agents such as Mycoplasma pneumoniae, Chlamydophila pneumoniae and Legionella pneumophila. In pediatric patients, the clinical and epidemiological aspects of M. pneumoniae

${ }^{1}$ Laboratorio de Microbiología Molecular, Instituto Nacional de Pediatría.

${ }^{2}$ Departamento de Infectología Pediátrica, Instituto Nacional de Pediatría.

${ }^{3}$ Laboratorio de Producción y Control de Biológicos. Escuela Nacional de Ciencias Biológicas, Instituto Politécnico Nacional.

Recibido: 21 de junio del 2017

Aceptado: 7 de septiembre del 2017

\section{Correspondencia}

De Colsa-Ranero A

agustin.decolsa@infecto.mx

Este artículo debe citarse como

Merida-Vieyra J, Aquino-Andrade A, Ribas-Aparicio RM, De Colsa-Ranero A. Cambiando los paradigmas de la infección por M. pneumoniae en pediatría. Acta Pediatr Mex. 2017;38(6):412-426. 
pneumonia need special attention, because in some cases do not have benign courses and sometimes they have extrapulmonary manifestations. The diagnostic approach is complex and doing a differential diagnosis from other etiological agents is complicated. Recently, the emergence of macrolide resistance in some countries has been alarming. There are controversies regarding antibiotic treatment. Worldwide, several studies, particularly from Asian countries, had shown new data regarding the biological, clinical, epidemiological, radiological and therapeutic response of $M$. pneumoniae in pediatric patients.

KEYWORDS: Mycoplasma pneumoniae; paediatric patients; pneumonia; atypical pneumonia
Correspondence

De Colsa-Ranero A

agustin.decolsa@infecto.mx

\section{INTRODUCCIÓN}

Mycoplasma pneumoniae es el microorganismo vivo más pequeño que infecta al humano. Clásicamente se había asociado al desarrollo de neumonía atípica; sin embargo, con la disponibilidad de métodos moleculares, se conoce que participa como colonizador de la vía aérea, pero también como patógeno de numerosos cuadros tanto pulmonares como extrapulmonares, siendo los cuadros más frecuente los neurológicos, hematológicos y cutáneos. Epidemiológicamente estaba asociado a cuadros principalmente en los adolescentes y adultos, en la actualidad se sabe que puede infectar a niños menores de 5 años, incluso condicionando cuadros graves y mortales. Su diagnóstico a través de laboratorio no está disponible en la mayoría de los centros hospitalarios y la interpretación de resultados puede ser compleja. En las últimas décadas se ha reportado un importante incremento en la resistencia de M. pneumoniae a los macrólidos, que son el tratamiento de elección en pediatría. Es importante que el clínico, principalmente el pediatra, conozca la epidemiología, el papel patogénico, la presentación clínica, la metodología diagnóstica disponible y los aspectos terapéuticos de $M$. pneumoniae.

\section{OBJETIVO}

Actualizar al médico pediatra en los recientes aspectos epidemiológicos, microbiológicos, clínicos, de laboratorio e imagen, así como consideraciones terapéuticas en las infecciones respiratorias por Mycoplasma pneumoniae en pacientes pediátricos.

\section{GENERALIDADES}

M. pneumoniae pertenece al género Mycoplasma, clase Mollicutes, en la cual se incluyen cuatro órdenes, cinco familias, ocho géneros y cerca de 200 especies que se han aislado de humanos, animales vertebrados, artrópodos y plantas. Entre estas especies se han descrito cinco más de importancia clínica: $M$. hominis, M. genitalium, M. fermentans, Ureaplasma urealyticum y $U$. parvum, que ocasionan enfermedades al ser humano, ya sea como patógeno principal u oportunista. ${ }^{1}$

M. pneumoniae es la bacteria más pequeña que existe. Su tamaño oscila entre $1 \mu \mathrm{m}$ de longitud y 0.1-0.2 um de ancho, representa apenas el 5\% de una bacteria típica (Escherichia coli). No es posible observarlo al microscopio óptico y no 
produce turbidez visible en medios de cultivo líquidos. Esta bacteria carece de pared celular, por lo tanto, no puede teñirse por la técnica de Gram y es resistente a la acción de los antibióticos betalactámicos. No es posible clasificarla como coco o bacilo debido a su pleomorfismo. No se encuentra de forma libre en la naturaleza debido a su total dependencia de un huésped que suplemente los nutrientes esenciales. ${ }^{2}$

\section{EPIDEMIOLOGÍA}

M. pneumoniae es un patógeno que infecta exclusivamente a humanos y es causante de infecciones del tracto respiratorio superior e inferior. Afecta principalmente a pacientes entre 5-20 años, pero puede presentarse en personas de todas las edades. ${ }^{3}$

Diferentes estudios han demostrado que de los pacientes con neumonía adquirida en la comunidad causada por $M$. pneumoniae, entre un 20 a $70 \%$ son menores de cinco años. ${ }^{4-8}$

Suele ser endémico, presentándose ciclos epidémicos cada 3-7 años. ${ }^{3}$ La mayoría de los brotes se presentan en comunidades cerradas, como bases militares, escuelas, campamentos de verano, comunidades religiosas y hospitales. Se ha descrito que parece no haber relación entre el clima, estación del año y ubicación geográfica con la infección por M. pneumoniae, por lo que se considera de distribución universal. Sin embargo, en los EE.UU. la mayoría de los brotes han ocurrido a finales del verano y principios del otoño. ${ }^{9} \mathrm{En}$ Japón se ha reportado una correlación entre el incremento de temperatura y las infecciones por M. pneumoniae, lo que puede explicar el incremento de casos en los meses cálidos del año. ${ }^{10}$ En el mundo se reportan frecuencias que van desde 0.74 hasta $43.8 \%$ (Figura 1). ${ }^{11-36}$

La aparición de ciclos epidémicos puede deberse a la circulación de diferentes genotipos de
M. pneumoniae (variación en la adhesina P1). En Japón, entre 1995 y 2001, M. pneumoniae tipo 2 fue el principal causante de infecciones; sin embargo, para el periodo 2002-2005 el tipo 1 fue el más prevalente. ${ }^{37}$ En Rusia, en el 2013, se presentó un brote por $M$. pneumoniae tipo 2c que incluyó 26 pacientes pediátricos y sus contactos. ${ }^{38}$ Esta variación en la circulación de diferentes tipos de $M$. pneumoniae impide el desarrollo de una respuesta inmune protectora en la población.

El periodo de incubación de $M$. pneumoniae es de 2-3 semanas, con un rango de 1-4 semanas. M. pneumoniae puede excretarse desde 2-8 días previos a la sintomatología, alcanzando un pico de excreción de 7-10 días y se extiende a 4-6 semanas o más. Se estima que alrededor del 3-10\% de los niños con infección por M. pneumoniae desarrollarán neumonía, y de estos, menos del 5\% necesitarán hospitalización. ${ }^{39}$ De los pacientes hospitalizados, hasta un $10 \%$ ingresa a unidad de cuidados intensivos ( $\mathrm{UCI}) .^{40}$

\section{PORTADORES ASINTOMÁTICOS}

M. pneumoniae puede estar presente en el tracto respiratorio de portadores asintomáticos. En Holanda se detectó a M. pneumoniae en el 21\% de niños sanos sin síntomas respiratorios. En este mismo estudio se observó que $M$. pneumoniae puede estar presente en el tracto respiratorio sin causar enfermedad hasta por un periodo de cuatro meses. ${ }^{41}$

En un estudio realizado en EE.UU. se reportó el $56 \%$ de niños sanos con $M$. pneumoniae y se describieron frecuencias de portadores del 6.7 al $13 \%$ durante la investigación de un brote. Debido a una respuesta inmune protectora de corta duración, M. pneumoniae puede persistir en el organismo después de la resolución de los síntomas. Además, los antibióticos utilizados en el tratamiento (macrólidos y tetraciclinas) son 


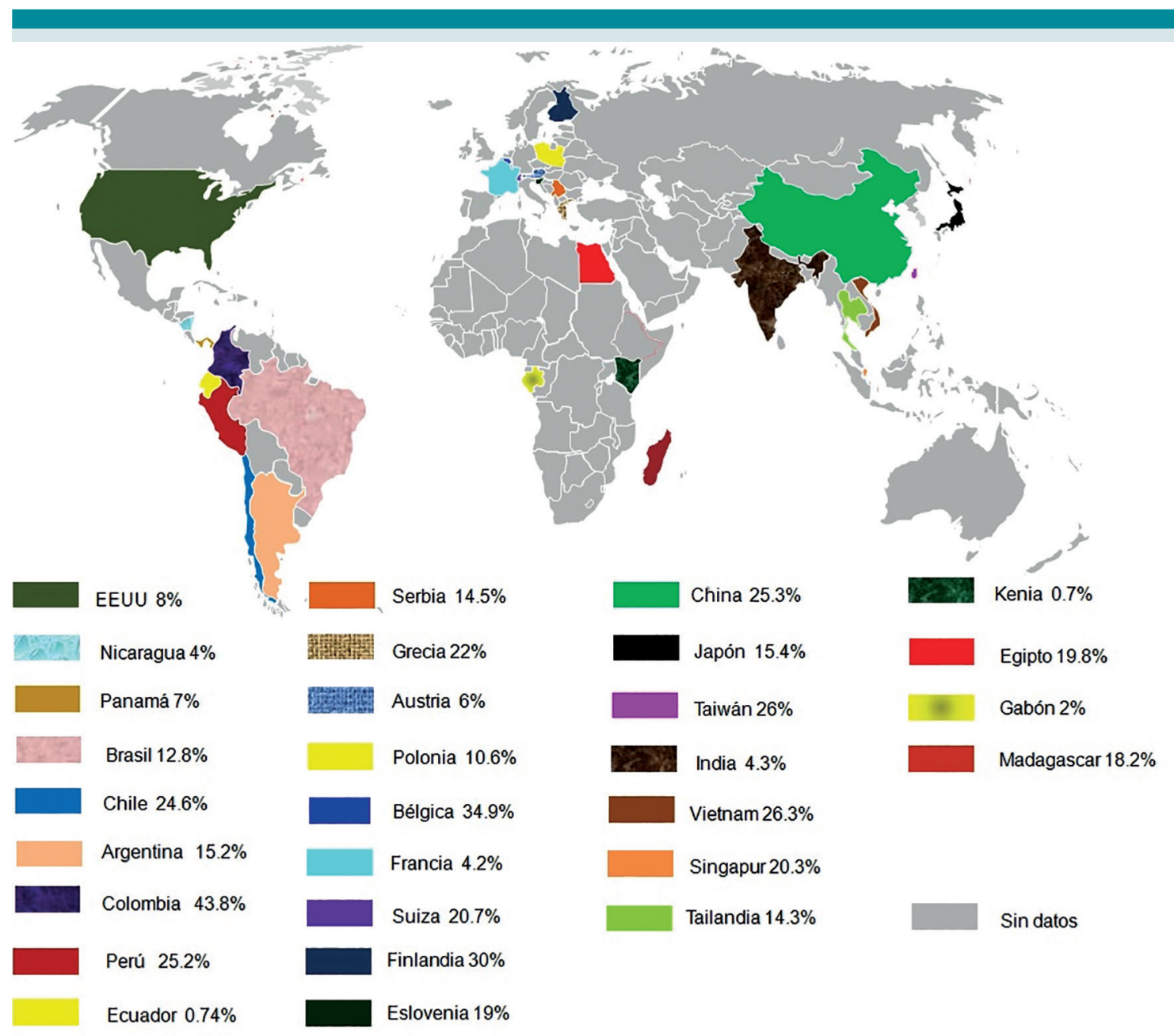

Figura 1. Epidemiología de M. pneumoniae.

Se muestra la prevalencia de este microorganismo en pacientes pediátricos alrededor del mundo. En México no existe información que documente la implicación de M. pneumoniae como agente causal de neumonía. . $^{6-8,11-36}$

agentes bacteriostáticos, lo que puede contribuir al estado de portador. ${ }^{10}$

\section{FISIOPATOLOGÍA}

Los principales mecanismos de patogenicidad de M. pneumoniae los podemos clasificar en: mecanismos de citoadherencia y mecanismos de citotoxicidad e inflamación.

\section{Mecanismos de citoadherencia}

M. pneumoniae infecta predominantemente el epitelio columnar ciliado respiratorio de la mucosa de todo el tracto respiratorio, para ello utiliza un organelo muy especializado que consiste en una serie de proteínas de adhesión, así como algunas otras accesorias de citoadhesión, las cuales son su principal factor de virulencia ya 
que las bacterias que pierden esta característica de adherencia son avirulentas. La adhesina P1 es el principal factor de adherencia, es una proteína transmembranal que se encuentra en la punta del organelo de adhesión, además de favorecer la citoadherencia al epitelio respiratorio favorece la movilidad de M. pneumoniae por deslizamiento. ${ }^{42}$

La segunda adhesina más importante es la P30, una proteína transmembranal, que se encuentra en forma de aglomerados en la punta del organelo de adhesión, también está involucrada en la movilidad por deslizamiento y coordina la división celular. Existen diversas proteínas accesorias de adhesión como las proteínas de alto peso molecular (HMW-1, HMW-2 y HMW-3); proteína A, proteína B y proteína-C, P65 y P116; todas ellas completan la adhesión del organelo al epitelio respiratorio. Estas proteínas pueden ser blancos terapéuticos e incluso ser antígenos para la elaboración de vacunas. La fusión de M. pneumoniae a las membranas de las células del epitelio respiratorio, podrían ser un factor crucial en la internalización de este patógeno, esto explicaría las infecciones latentes e incluso crónicas, por la evasión del sistema inmune. ${ }^{42}$ Al parecer, la gravedad de la enfermedad tiene una relación directa con el daño a nivel ciliar. ${ }^{43}$

\section{Mecanismos de citotoxicidad e inflamación}

Debido a que M. pneumoniae carece de pared celular, no contiene antígenos estimulantes, como es el lipopolisacárido capsular, peptidoglicanos o ácido teicoico. M. pneumoniae causa daño a través de dos vías: la primera, el estrés oxidativo por la formación de radicales libres como el peróxido de hidrógeno y el superóxido que dañan al epitelio y los cilios, y la segunda es la respuesta inflamatoria condicionada por la cascada de las citoquinas proinflamatorias. ${ }^{44}$

M. pneumoniae activa los receptores de patrones de reconocimiento (PRR) a través de diferentes receptores Toll-like (TLR-1, TLR-2, TLR-6), activando el factor de transcripción nuclear $k \beta(N F-k \beta)$, que es un regulador clave en la activación de la cascada proinflamatoria, principalmente a través de la producción de IL-1 $\beta$, IL-8 y TNF- $\alpha$. Se han reportado niveles elevados de IL-18 en la fase aguda de la neumonía por M. pneumoniae, sugiriendo el rol de citoquinas vía Th1; sin embargo, otros reportes describen una polarización hacia Th2. ${ }^{45}$

La denominada toxina CARDS (acrónimo por sus siglas en inglés de Community Acquired Respiratory Distress Syndrome) es una toxina vacuolizante y ribosilante de ADP; presenta homología con la subunidad S1 de la toxina pertussis, la cual tiene una alta afinidad al surfactante-A a nivel alveolar. ${ }^{46}$ En modelos animales induce una respuesta proinflamatoria en el tracto respiratorio, estimula la producción de IL-1, IL-6, IL-12 y TNF- $\alpha$ e incrementa la expresión de la respuesta inflamatoria vía Th2, con producción predominante de las citoquinas IL-4, IL-3 y de las quimoquinas CCL-7 y CCL-32. También la toxina CARDS produce inflamación a través de la regulación del inflamosoma, que es un complejo multiprotéico localizado a nivel citoplasmático y es el responsable de la activación de la enzima caspasa-1, la cual a su vez activa el paso de pro-IL- $1 \beta$ a IL- $1 \beta .{ }^{42,46}$

También se han reconocido más de 30 lipoproteínas de M. pneumoniae que poseen potentes propiedades inflamatorias, como son la $\mathrm{N}$ ALP1/N-ALP2 y la FOF1-ATPasa, que también activan el NF-k $\beta$, vía TLR-1, TLR-2 y TLR-6, que ocasionan movilización y reclutamiento de linfocitos y neutrófilos a nivel pulmonar, produciendo inflamación. ${ }^{45}$

Desde el punto de vista histopatológico hay ulceración y descamación de la mucosa respiratoria, con destrucción del epitelio ciliado. Se produce un exudado inflamatorio que contiene 
fibrina, infiltrado mononuclear con linfocitos y monocitos, así como la presencia de polimorfonucleares. A nivel alveolar hay edema con infiltración de linfocitos, células mononucleares, células plasmáticas e incluso eritrocitos. Este edema e infiltrado celular suelen extenderse al espacio intersticial, observándose regiones con franca congestión y hemorragia. ${ }^{47}$

La enfermedad causada por M. pneumoniae se da por invasión directa o por reacción inmunológica. A través de estudios de PCR se ha detectado el DNA de M. pneumoniae en diversos líquidos y tejidos: sangre, líquido pleural, líquido pericárdico, líquido articular y líquido cefalorraquídeo; así como de oído medio, riñón, cerebro, piel (en lesiones cutáneas), hígado, bazo y ganglios, entre otros. ${ }^{47}$

\section{INMUNIDAD CONTRA M. pneumoniae}

La respuesta inmune contra $M$. pneumoniae depende del estado inmunológico del huésped, impactando directamente en la gravedad del cuadro y la aparición de manifestaciones extrapulmonares. La infección por M. pneumoniae produce respuesta innata involucrando macrófagos, mastocitos, neutrófilos y células NK, así como una respuesta adaptativa con anticuerpos tipo IgA, IgM e $\operatorname{lgG} .^{48}$ Además, hay una adecuada respuesta celular con linfocitos $\mathrm{B}$ y $\mathrm{T}$. Al parecer, la respuesta humoral no condiciona protección contra reinfecciones; sin embargo, el efecto neutralizante de los anticuerpos podría ser importante para la contención en la infección aguda, ya que pacientes con deficiencias humorales pueden cursar con cuadros más graves o prolongados, además de tener más riesgo para manifestaciones extrapulmonares, particularmente a nivel neurológico. ${ }^{10}$

El papel de los macrófagos parece ser vital en controlar la infección a nivel pulmonar, ya que interviene en la opsonización y depuración del patógeno. La activación de macrófagos depende de los receptores Toll-like, particularmente el TLR2. ${ }^{49}$ En relación al perfil de citoquinas, no hay una clara polarización hacia una respuesta Th1 o Th2; ${ }^{50}$ por una parte predomina la producción de IL-8 e IL-18, en donde la IL-8 es un potente reclutador de neutrófilos, mientras que la IL-18 se encarga de activar a los linfocitos T. Los mastocitos a su vez son productores de IL-4/IF- $\gamma$, siendo inductor de la producción de citoquinas por los macrófagos, leucocitos periféricos y a nivel del epitelio respiratorio. ${ }^{10}$ La producción de citoquinas y la activación linfocítica por una parte puede eliminar a M. pneumoniae, o bien, causar exacerbaciones por hipersensibilidad inmunológica, agravando el daño del epitelio respiratorio y espacio alveolar.

\section{MANIFESTACIONES CLÍNICAS}

El término de "neumonía atípica" fue acuñado desde la década de 1930, incluso antes de que se determinara su etiología; se le designó de esa manera ya que contrastaba con los datos de neumonía más grave como eran fiebre alta, importante ataque al estado general y estado tóxico, que se observaban clásicamente con las neumonías por agentes piógenos como Streptococcus pneumoniae y Staphylococcus aureus. Más adelante también se le llamó la "neumonía del caminante", debido a la poca afección sistémica aparente y relativo buen estado general del paciente. ${ }^{45}$

M. pneumoniae causa infecciones del tracto respiratorio tanto superior como inferior siendo la neumonía el cuadro de mayor impacto clínico y epidemiológico. La mayoría de las infecciones por M. pneumoniae generalmente son leves y auto limitadas; sin embargo, en la actualidad se reconoce que puede ocasionar cuadros graves y refractarios como neumonía fulminante, neumonía necrotizante y desarrollo del síndrome de dificultad respiratoria del 
adulto (SDRA). ${ }^{51}$ También se ha asociado a otras infecciones del tracto respiratorio superior como: faringitis, bronquitis, laringotraqueítis, rinosinusitis, otitis media, miringitis y bronquiolitis. ${ }^{52}$ Además, causa una gran variedad de infecciones extrapulmonares que afectan prácticamente a cualquier órgano. ${ }^{53}$

Habitualmente, el cuadro clínico por M. pneumoniae inicia como una infección de vía aérea superior que suele ser similar a la de un cuadro viral; sin embargo, son poco frecuentes los datos de rinitis, sinusitis y laringotraqueobronquitis. ${ }^{10,53}$ La presencia de fiebre se ha documentado entre un $38-100 \%{ }^{4,7,34}$ La faringitis por M. pneumoniae se reporta entre un $12-24 \%$ de las faringitis no-estreptocóccicas. ${ }^{4,54}$

La afección bronquial y traqueobronquial es muy frecuente, observándose en un $70-80 \%$ de los casos, es por ello que la tos no productiva y prolongada caracteriza estas infecciones. La linfadenopatía no es un hallazgo frecuente. La exploración pulmonar puede ser incluso normal o haber hipoventilación. En el $60 \%$ de los casos no se documentan estertores, mientras que $33 \%$ pueden tener estertores tardíos de despegamiento. 4,55

En relación a la presencia de sibilancias existen controversias, algunos estudios muestran que se presentan en $20-64 \%$ de los $\operatorname{casos}^{4,31}$ y por otra parte existen reportes que señalan que la ausencia de sibilancias es más compatible con infecciones por M. pneumoniae.

En una revisión que agrupó siete estudios e incluyó 1,491 niños y adolescentes, se evidencia que no puede hacerse el diagnóstico de $M$. pneumoniae basado únicamente en el contexto clínico. En dos de estos estudios se encontró que el dolor torácico es más frecuente en neumonías con M. pneumoniae; sin embargo, este es un dato que no puede ser referido por niños menores. ${ }^{56}$ Lo mismo pasa con la cefalea, que al parecer ha resultado ser más frecuente en las infecciones por $M$. pneumoniae, ${ }^{34} \sin$ embargo, niños menores pueden no manifestarla tan claramente. También se ha documentado que los pacientes con M. pneumoniae pueden cursar con vómito (13-28\%), dolor abdominal en 15\% y diarrea hasta un $10 \%{ }^{7,34} \mathrm{~A}$ pesar de contar con esta variedad de signos y síntomas, basándose exclusivamente en la presentación clínica no pueden identificarse los cuadros neumónicos por M. pneumoniae y diferenciarlos de otros agentes como virus o bacterias piógenas. ${ }^{4,10,31}$

Existe una asociación entre la infección por M. pneumoniae y asma; sin embargo, los diferentes estudios demuestran ciertas discordancias en la participación de este patógeno, encontrándose tasas tan bajas como 3-6.6\% en niños hospitalizados por asma y en otro extremo tasas tan elevadas como $29 \%$ en niños con cuadros iniciales con sibilancias y hasta $48-64 \%$ en niños con sibilancias recurrentes. ${ }^{31}$ Uno de los estudios más extensos buscando la asociación de asma e infección por M. pneumoniae se llevó a cabo en Taiwán, en donde se reclutaron 1,591 niños con infección por M. pneumoniae comparados con 6,364 controles, en este estudio se encontró un mayor desarrollo de asma cuando se cursaba con infección por M. pneumoniae. ${ }^{57}$

Los cuadros graves y fatales suelen ocurrir principalmente en pacientes jóvenes y adultos, la neumonía fatal por $M$. pneumoniae se estima que es entre 0.5 y $2 \%$ de los casos; sin embargo, hay estudios en los que también está reportado que los cuadros graves se presentan con mayor frecuencia en pacientes menores de 5 años. El derrame pleural se observa entre el 10-34\%. ${ }^{7} \mathrm{Si}$ bien la mayoría de las muertes se asocia a cuadros respiratorios como neumonía fulminante, neumonía necrotizante y desarrollo de SDRA, también se han reportado cuadros fatales sin componente neumónico, como puede ser la miocarditis, encefalitis, tromboembolismo pulmonar, entre otras. ${ }^{58}$ 


\section{COMPLICACIONES EXTRAPULMONARES}

Las infecciones respiratorias causadas por M. pneumoniae se pueden complicar con manifestaciones extrapulmonares hasta en un $25 \%$ de los casos, afectando principalmente sistema nervioso, piel y mucosas, sistema hematológico, sistema cardiovascular, sistema osteoarticular, entre otros. Estas complicaciones pueden incluso ocurrir aún sin la presencia de neumonía. ${ }^{42}$ Las complicaciones extrapulmonares pueden clasificarse de acuerdo a los tres posibles mecanismos de patogenicidad de M. pneumoniae: ${ }^{53}$ invasión directa de la bacteria al sitio anatómico específico, generando una respuesta inflamatoria local; lesión indirecta en donde la bacteria no está presente, pero se ha desencadenado una respuesta inmunológica contra la bacteria, generando complejos inmunes o autoinmunidad, y oclusión vascular que ocasiona isquemia, derivada de una lesión directa o indirecta por M. pneumoniae.

En el Cuadro 1 se muestran las diferentes complicaciones extrapulmonares ocasionadas por M. pneumoniae. ${ }^{53,59}$

\section{COINFECCIÓN CON OTROS PATÓGENOS}

La participación de otros patógenos durante la infección de M. pneumoniae ha sido ampliamente discutida. Se ha descrito la coinfección entre un $50-90 \% .{ }^{40,60}$ En un estudio multicéntrico en EE.UU., que reunió 171 pacientes pediátricos con infección por M. pneumoniae, se documentó que en un 65\% de los pacientes existía coinfección con otros patógenos, siendo el 38\% de los casos con bacterias piógenas como Haemophilus influenzae, S. pneumoniae, S. aureus, Moraxella catarrhalis y Streptococcus pyogenes; mientras que los agentes virales se presentaron en un $7.6 \%$, siendo los rinovirus y los coronavirus los agentes más frecuentes. La coinfección mixta con virus y bacterias se documentó en un $20 \%{ }^{40}$
Cuadro 1. Complicaciones extrapulmonares por M. pneumoniae

\begin{tabular}{|c|c|}
\hline Órgano/sistema & Complicaciones \\
\hline Piel & $\begin{array}{l}\text { Síndrome de Stevens Johnson, mucositis, } \\
\text { exantema, eritema, dermatosis subcor- } \\
\text { neal, vasculitis, liquen plano, necrólisis } \\
\text { epidérmica tóxica, eritema multiforme, } \\
\text { urticaria, púrpura anafilactoide, eritema } \\
\text { nodoso, mucositis }\end{array}$ \\
\hline Neurológicas & $\begin{array}{l}\text { Meningoencefalitis, encefalitis/encefalitis } \\
\text { diseminada aguda, meningitis aséptica, } \\
\text { mielitis transversa, síndrome de Guillain } \\
\text { Barré/síndrome de Miller Fisher, neuro- } \\
\text { patía variante de Guillain Barré, neuro- } \\
\text { patías craneales/periféricas, cerebelitis } \\
\text { ataxia cerebral aguda, parkinsonismo } \\
\text { transitorio, síndrome de Opsoclonus- } \\
\text { Mioclonus, evento cerebral isquémico, } \\
\text { necrosis talámica/cuerpo estriado }\end{array}$ \\
\hline Hematológicas & $\begin{array}{l}\text { Trombocitopenia, púrpura de Henoch- } \\
\text { Schonlein, síndrome hemofagocítico, } \\
\text { síndrome de hipoprotrombinemia an- } \\
\text { ticoagulante lúpico, trombosis arterial, } \\
\text { rabdomiolisis, infarto esplénico, coagu- } \\
\text { lación intravascular diseminada (CID) }\end{array}$ \\
\hline Cardiacas & $\begin{array}{l}\text { Miocarditis, pericarditis, endocarditis, } \\
\text { enfermedad trombótica cardiaca/aórtica }\end{array}$ \\
\hline $\begin{array}{l}\text { Músculo- } \\
\text { esquelético }\end{array}$ & Artritis, rabdomiolisis \\
\hline $\begin{array}{l}\text { Órganos de los } \\
\text { sentidos }\end{array}$ & $\begin{array}{l}\text { Ojo: conjuntivitis, iritis, uveítis } \\
\text { Oído: otitis media, pérdida auditiva } \\
\text { súbita }\end{array}$ \\
\hline Digestivas & $\begin{array}{l}\text { Hepatitis/hepatitis fulminante, pan- } \\
\text { creatitis. }\end{array}$ \\
\hline Nefrourinario & $\begin{array}{l}\text { Glomerulonefritis, nefropatía por IgA, } \\
\text { embolismo de arteria renal, priapismo }\end{array}$ \\
\hline Misceláneas & $\begin{array}{l}\text { Enfermedad de Kawasaki mononucleosis } \\
\text { infecciosa }\end{array}$ \\
\hline
\end{tabular}

Es importante comentar que la codetección no implica coinfección a nivel pulmonar, muchos de estos microorganismos en tracto naso-orofaríngeo son considerados colonizantes, pero potencialmente patógenos; detectarlos en estos sitios no indica que sean responsables del cuadro infeccioso, por lo que el hallazgo puede ser clínicamente irrelevante. Esta asociación aún no es clara, por lo que se requieren estudios prospectivos con identificación de los diversos 
patógenos en lavados broncoalveolares en pacientes con neumonía.

\section{MÉTODOS DIAGNÓSTICOS}

\section{Estudios de imagen}

No existe un patrón radiológico característico en general que se correlacione con la información clínica. En las radiografías de tórax, lo descrito tradicionalmente son patrones intersticiales difusos bilaterales, opacidades reticulares, así como opacidades en parche, que cuando son bilaterales, suelen estar cerca del hilio o en lóbulos inferiores. Los infiltrados focales, como consolidación, el derrame pleural y adenopatía hiliar, se observan con menor frecuencia. ${ }^{61}$

En una extensa serie de 1,280 casos pediátricos con neumonía por $M$. pneumoniae, que fueron evaluados con TAC pulmonar, se documentó con mayor precisión que el $54 \%$ de las lesiones eran unilaterales, mientras que el $46 \%$ fueron bilaterales. Los patrones tomográficos fueron diversos, predominando las opacidades extensas en parche (86\%); con menos frecuencia los infiltrados en patrón moteado, así como imagen en vidrio despulido. Otro hallazgo significativo fue que en un $77 \%$ se encontró engrosamiento de las paredes bronquiales. El derrame pleural se documentó en un $11 \%$, mientras que la adenopatía mediastinal en un 10\%. ${ }^{61}$ También se ha encontrado que en cuadros más graves y refractarios se observan más las consolidaciones segmentaria-lobar (50-62\%), así como incremento en el derrame pleural hasta el $22 \% .^{51}$

\section{Biometría hemática}

Al parecer no tiene hallazgos específicos, no es frecuente el desarrollo de leucocitosis, y normalmente el recuento diferencial de leucocitos suelen no tener un predominio; incluso, los cuadros de neumonías graves y complicadas no han mostrado cambios significativos en los recuentos hemáticos. ${ }^{9}$ No obstante, si se ha re- portado elevación de la proteína $\mathrm{C}$ reactiva en cuadros refractarios. ${ }^{51}$

Algunos estudios han demostrado también que la $\mathrm{BH}$ no tiene diferencias significativas en comparación con neumonías ocasionadas por otros patógenos. ${ }^{34}$ Un hallazgo relevante es que se ha documentado que la elevación de la deshidrogenasa láctica $(\mathrm{DHL})$ puede ser un biomarcador predictivo de la gravedad de la neumonía por M. pneumoniae, ya que en pacientes tanto pediátricos como adultos los niveles de DHL se correlacionan con el desarrollo de neumonía fulminante. ${ }^{58,62}$

\section{Serología}

Las pruebas serológicas incluyen crioaglutininas, fijación de complemento y ensayos inmunoenzimáticos; estos últimos, es el más utilizado para el diagnóstico de M. pneumoniae.

\section{a) Crioaglutininas}

La formación de crioaglutininas es la primera respuesta humoral que se observa frente a la infección por M. pneumoniae. Aparecen entre la primera y segunda semana y desaparecen de dos a tres meses después. No son un buen indicador de infección, debido a que solo se presentan en el 50-60\% de los casos. Los resultados falso-positivos son frecuentes; se observan reacciones cruzadas con el virus Epstein-Barr, Citomegalovirus, Klebsiella pneumoniae, Treponema pallidum, virus de la influenza, Adenovirus y L. pneumophila. ${ }^{63}$

\section{b) Inmunoensayo enzimático (ELISA)}

El ELISA es el método inmunológico más utilizado para el diagnóstico de infección por M. pneumoniae. Permite la detección de IgM, $\lg$ e IgA. Tiene una mayor sensibilidad, en comparación con el cultivo, para detectar la infección por M. pneumoniae en la fase aguda. Además, tiene la ventaja de ser cualitativa o 
cuantitativa, no requerir equipo especializado y utilizar pequeños volúmenes de suero. ${ }^{64} \mathrm{La}$ lgM aparece entre 7-10 días después del inicio de la infección y sus niveles son más altos en la primera infección que en las subsecuentes. Los niveles de $\lg \mathrm{G}$ son detectables aproximadamente en la quinta semana y pueden persistir por varios años después de una infección aguda. ${ }^{8}$ Para un diagnóstico adecuado es necesario colectar suero en la fase aguda y en la fase de convalecencia. Un incremento de cuatro veces o más en el título de anticuerpos indica una infección reciente. ${ }^{65}$

El inconveniente de utilizar IgM como marcador de infección aguda en niños radica en que pacientes menores de 6 meses pueden no producir IgM. Otra importante limitación de la serología para el diagnóstico de infección aguda por M. pneumoniae radica en una inadecuada producción de anticuerpos en algunas infecciones o una respuesta inmune humoral deficiente como resultado de alguna inmunosupresión. Resultados falso-negativos pueden ocurrir si el suero es colectado después de la administración del tratamiento con antimicrobianos. ${ }^{66}$

\section{Diagnóstico de microbiológico}

El diagnóstico de la infección por M. pneumoniae es difícil, debido a que es un microorganismo de crecimiento lento (difícil cultivo), la considerable seroprevalencia entre la población y la presencia de portadores asintomáticos. ${ }^{67}$ Este diagnóstico se basa principalmente en cultivo y detección de ácidos nucleicos.

\section{a) Cultivo}

El cultivo es considerado el estándar de oro para el diagnóstico de infección por M. pneumoniae. Las muestras disponibles para cultivo incluyen exudado faríngeo, esputo, aspirado nasal o traqueal, lavado bronquioalveolar o líquido pleural. ${ }^{52}$ Se recomienda utilizar un medio de transporte adecuado, como caldo soya tripticaseína con $0.5 \%$ de albumina sérica bovina para evitar la desecación. El tiempo entre la toma de muestra y el envío al laboratorio no debe ser mayor a cuatro horas. Si no es posible el transporte inmediato al laboratorio, las muestras pueden almacenarse a $4^{\circ} \mathrm{C}$. Sin embargo, el crecimiento de M. pneumoniae es lento (de tres a seis semanas) y difícil debido a sus altos requerimientos nutricionales (adición de suero al medio de cultivo) por lo que no es recomendable para el diagnóstico de rutina. El cultivo se reserva para la tipificación molecular de los aislamientos y realizar pruebas de sensibilidad a antimicrobianos. ${ }^{65}$

\section{b. Detección de ácidos nucleicos}

El primer reporte de la PCR (por sus siglas en inglés polymerase chain reaction) para la detección de $M$. pneumoniae en muestras clínicas fue en 1989. Desde entonces se han publicado varios reportes que utilizan diferentes blancos genéticos como el gen que codifica al 16S rRNA, el gen $p 1$, el operón de la ATPasa, tuf, parE, dnaK, pdhA, repMp1 y el gen CARDS.9,68 Las ventajas de la PCR sobre el cultivo y la serología radican en una mayor sensibilidad, completar el proceso de la muestra en un solo día, la obtención de un resultado positivo en menor tiempo en comparación con la serología y el requerimiento de una sola muestra en la cual $M$. pneumoniae no necesariamente debe estar viable. ${ }^{66}$ Las muestras clínicas disponibles para la prueba de PCR incluyen secreciones orofaríngeas y nasofaríngeas, esputo, lavado traqueobronquial y biopsia de pulmón. ${ }^{65}$ Se ha sugerido que el uso de la PCR en combinación con la serología puede proporcionar un mejor diagnóstico y puede ayudar a distinguir entre colonización e infección verdadera. ${ }^{9}$

\section{TRATAMIENTO}

Los antibióticos con actividad contra M. pneumoniae son los macrólidos, los ketólidos, las 
tetracilinas y las fluoroquinolonas. ${ }^{69}$ En adultos, los macrólidos, las fluoroquinolonas y las tetraciclinas son los tratamientos de elección y en pacientes pediátricos únicamente los macrólidos. Aún es desconocido el valor terapéutico del uso de antibióticos en niños hospitalizados con neumonía por M. pneumoniae. En la actualidad se cuestiona la verdadera utilidad de los antibióticos para el tratamiento de M. pneumoniae. ${ }^{70}$

Una revisión sistemática de 17 estudios no encontró significancia estadística en el beneficio del uso de antibióticos en aquellos pacientes con infección respiratoria baja por M. pneumoniae. ${ }^{71}$ De manera similar, en una revisión que involucró siete estudios con 1,912 niños con infección del tracto respiratorio inferior por M. pneumoniae, se analizó la respuesta clínica de pacientes tratados con esquemas de antibióticos basados en macrólidos, comparada con esquemas que no incluían macrólidos, encontrándose que no había ninguna diferencia. ${ }^{72}$ En contraparte, hay estudios que reflejan que pacientes con $M$. pneumoniae sensibles a macrólidos (MPSM), al ser tratados con los mismos, tienen una mejor evolución clínica y menor índice de complicaciones que aqueIlos pacientes con $M$. pneumoniae resistentes a macrólidos (MPRM). ${ }^{73,74}$ Estas evidencias nos permiten replantearnos que si el uso de macrólidos no fuera de utilidad, los cursos clínicos tanto de infecciones por MPSM como MPRM deberán ser similares; precisamente en estos estudios comparativos se vislumbra el posible beneficio de los macrólidos. En la actualidad, aunque no existe una evidencia sólida que nos indique que sea necesaria una terapéutica antimicrobiana contra M. pneumoniae, en la práctica clínica se continúa su recomendación. Para cuadros graves y refractarios está bien documentada la utilidad del tratamiento con corticoesteroides. ${ }^{58,62}$

\section{RESISTENCIA ANTIMICROBIANA}

Debido a que M. pneumoniae carece de pared celular es intrínsecamente resistente a los beta- lactámicos. Antes del año 2000 las infecciones por M. pneumoniae eran tratadas esencialmente con macrólidos, ya que los aislamientos clínicos con resistencia a estos antimicrobianos era muy rara. ${ }^{69}$

En el año 2001, se reportó en Japón la primera mutación en el gen $23 \mathrm{~S}$ rRNA en M. pneumoniae, en un paciente pediátrico con neumonía; a partir de entonces, la resistencia se ha ido extendiendo a nivel mundial. Los índices de resistencia a macrólidos varían de acuerdo a la región del mundo. En Asia, se reportan frecuencias de hasta el $90 \%$, en Europa del $1-26 \%$, mientras que en EE.UU. y Canadá se encuentran en $13 \%$ y $12 \%$, respectivamente. ${ }^{39,40}$ Esto constituye un problema en el tratamiento de infecciones en pacientes pediátricos debido a que las alternativas terapéuticas como tetraciclinas, no son recomendadas para niños. ${ }^{75}$

El mecanismo molecular de la resistencia se basa en mutaciones puntuales en el dominio $\mathrm{V}$ de la subunidad 23S del rRNA (posiciones 2058, 2059, 2062, 2611), lo que reduce la afinidad del antibiótico al ribosoma ${ }^{69}$ y ocasionalmente también involucra mutaciones en las proteínas ribosomales L4 y L22. ${ }^{69,76}$

Existe la evidencia de que las cepas de M. pneumoniae con resistencia a macrólidos pueden cursar con cuadros más graves y con mayor frecuencia manifestaciones extrapulmonares. ${ }^{74} \mathrm{En}$ pacientes con cepas resistentes, las alternativas terapéuticas son quinolonas y en niños mayores de 8 años las tetraciclinas como la minociclina. ${ }^{77}$ A la fecha, no hay evidencia de resistencia a las fluoroquinolonas o a las tetraciclinas. ${ }^{69}$ En la actualidad, se estudian nuevos antimicrobianos potencialmente útiles contra cepas resistentes a macrólidos como: la lefamulina (pleuromutilina), solitromicina y nafitromicina (ketólidos), omadaciclina (aminometilciclina) y la zoliflodacina (espiropirimidinetriona). ${ }^{10}$ 


\section{VACUNAS CONTRA M. pneUmoniae}

Las vacunas contra $M$. pneumoniae datan de 1965, originalmente se probaron vacunas inactivadas, documentándose adecuada producción de anticuerpos; sin embargo, se presentaron efectos adversos en algunos grupos de pacientes que inicialmente no habían elevado anticuerpos, pero cuando posteriormente cursaban con infección por M. pneumoniae tenían cuadros más severos, lo que indicaba una posible sensibilización con la inmunización primaria. ${ }^{78}$ También se han utilizado vacunas vivas atenuadas; no obstante, las reinfecciones por M. pneumoniae eran frecuentes, por lo que no parecieron ser tan efectivas. Recientemente, se han desarrollado estrategias con vacunas basadas en antígenos, administrándose tanto a nivel intranasal como sistémicas, utilizando proteínas recombinantes inmunogénicas como la proteína de la adhesina $\mathrm{P} 1$, específicamente la parte C-terminal de la misma (RP14), induciendo una inmunogenicidad sólida tanto a nivel de mucosas como sistémica. También se han utilizado proteínas quiméricas de RP14 y la proteína de adhesión P30, que inducen una adecuada respuesta con producción IgA a nivel de mucosas, que además de prevenir infecciones, pudiera incluso tener un efecto para evitar la colonización. ${ }^{79}$

\section{CONCLUSIONES}

M. pneumoniae tradicionalmente se había considerado como agente de neumonía atípica en adolescentes y adultos, aparentemente con cursos benignos e incluso auto limitados. En la última década, ha surgido mucha información en relación a M. pneumoniae en pacientes pediátricos, por lo que empieza a haber un cambio de paradigma en los conceptos conocidos por este patógeno. En la actualidad M. pneumoniae ya se reconoce como un colonizante de la vía aérea y sabemos que no es un patógeno infrecuente como causa de neumonía, además de que no es exclusivo del adolescente y adulto, sino que incluso puede afectar niños menores de 5 años. Si bien los cursos suelen ser benignos, hay cuadros graves y complicados. M. pneumoniae cada vez empieza a tomar un papel fundamental en cuadros extrapulmonares. El diagnóstico clínico no suele cursar con datos específicos, y puede ser indistinguible de neumonías virales y bacterianas, lo que hace indispensable su evaluación por laboratorio tanto con pruebas serológicas y moleculares. Finalmente, aunque aún no queda establecido el real valor terapéutico de los antibióticos en el curso de la enfermedad, en la actualidad se siguen recomendando. En esta nueva era en que la resistencia a macrólidos es emergente, otros antibióticos aparecen como alternativas. Sin duda, se requieren más estudios prospectivos para acabar de resolver aún de los muchos interrogantes en relación a este patógeno.

\section{REFERENCIAS}

1. Waites KB, Xiao L, Paralanov V, Viscardi RM, Glass JI. Mycoplasma and Ureaplasma. Mol Typing Bact Infect. Primera. New York: Springer Science+Business Media; 2013. p. 229-281.

2. Waites KB, Simecka JW, Talkington DF, Prescott T. Pathogenesis of Mycoplasma pneumoniae infections: adaptive immunity, innate immunity, cell biology, and virulence factors. Community Acquir-Pneumonia. Switzerland: Birkhäuser Verlag Basel; 2007. p. 183-199.

3. Matas Andreu L, Molinos Abós S, Fernández Rivas G, González Soler V, Ausina Ruiz V. Serologic diagnosis of Mycoplasma pneumoniae infections. Enferm Infecc Microbiol Clin. 2006;24(1):19-23.

4. He X, Wang X, Zhang R, Yuan Z, Tan J, Peng B, et al. Investigation of Mycoplasma pneumoniae infection in pediatric population from 12,025 cases with respiratory infection. Diagn Microbiol Infect Dis. 2013;75(1):22-27.

5. Diaz MH, Benitez AJ, Cross KE, Hicks LA, Kutty P, Bramley $\mathrm{AM}$, et al. Molecular Detection and Characterization of Mycoplasma pneumoniae Among Patients Hospitalized With Community-Acquired Pneumonia in the United States. Open Forum Infect Dis. 2015;2(3):ofv106.

6. Chiang WC, Teoh OH, Chong CY, Goh A, Tang JPL, Chay OM. Epidemiology, clinical characteristics and antimicrobial resistance patterns of community-acquired pneumonia in 
1702 hospitalized children in Singapore. Respirol Carlton Vic. 2007;12(2):254-261.

7. Ma Y-J, Wang S-M, Cho Y-H, Shen C-F, Liu C-C, Chi H, et al. Clinical and epidemiological characteristics in children with community-acquired mycoplasma pneumonia in Taiwan: A nationwide surveillance. J Microbiol Immunol Infect. 2015;48(6):632-638.

8. Vervloet LA, Vervloet VEC, Tironi Junior M, Ribeiro JD. Mycoplasma pneumoniae-related community-acquired pneumonia and parapneumonic pleural effusion in children and adolescents. J Bras Pneumol. 2012;38(2):226-236.

9. Waites KB. ¿What's new in diagnostic testing and treatment approaches for Mycoplasma pneumoniae infections in children? Hot Top Infect Immun Child V. New York: Springer; 2011. p. 45-57.

10. Waites KB, Xiao L, Liu Y, Balish MF, Atkinson TP. Mycoplasma pneumoniae from the Respiratory Tract and Beyond. Clin Microbiol Rev. 2017;30(3):747-809.

11. Almasri M, Papa A, Souliou E, Haidopoulou K, Eboriadou M. Respiratory syncytial virus infection in hospitalized children older than 2 years with community-acquired pneumonia. Hippokratia. 2013;17(2):146-149.

12. Cantais A, Mory O, Pillet S, Verhoeven PO, Bonneau J, Patural $\mathrm{H}$, et al. Epidemiology and microbiological investigations of community-acquired pneumonia in children admitted at the emergency department of a university hospital. J Clin Virol. 2014;60(4):402-407.

13. Carcey J, Garcia P, Padilla O, Castro-Rodriguez JA. Increased prevalence of Mycoplasma pneumoniae serological positivity in Chilean young children. Allergol Immunopathol (Madr). 2016;44(5):467-471.

14. De Schutter I, De Wachter E, Crokaert F, Verhaegen J, Soetens $\mathrm{O}$, Piérard D, et al. Microbiology of bronchoalveolar lavage fluid in children with acute nonresponding or recurrent community-acquired pneumonia: identification of nontypeable Haemophilus influenzae as a major pathogen. Clin Infect Dis. 2011;52(12):1437-1444.

15. Del Valle-Mendoza J, Orellana-Peralta F, Marcelo-Rodríguez A, Verne E, Esquivel-Vizcarra M, Silva-Caso W, et al. High Prevalence of Mycoplasma pneumoniae and Chlamydia pneumoniae in Children with Acute Respiratory Infections from Lima, Peru. PloS One. 2017;12(1):e0170787.

16. Feikin DR, Njenga MK, Bigogo G, Aura B, Aol G, Audi A, et al. Etiology and Incidence of viral and bacterial acute respiratory illness among older children and adults in rural western Kenya, 2007-2010. PloS One. 2012;7(8):e43656.

17. Ferrero FC, Ossorio MF, Eriksson PV, Duran AP. Mycoplasma pneumoniae en niños con neumonía. Arch Argent Pediatr. 2000;98(1):12-17.

18. Grassi T, Mancini F, Ciervo A, Vescio MF, Ghazal A, Ashour H, et al. Chlamydophila pneumoniae, Mycoplasma pneumoniae, and influenza in children with respiratory infections in Alexandria, Egypt. J Infect Dev Ctries. 2014;8(3):379-383.
19. Herrera M, Aguilar YA, Rueda ZV, Muskus C, Vélez LA. Comparison of serological methods with PCR-based methods for the diagnosis of community-acquired pneumonia caused by atypical bacteria. J Negat Results Biomed. 2016;15:3. doi.org/10.1186/s12952-016-0047-y

20. Huong PLT, Hien PT, Lan NTP, Binh TQ, Tuan DM, Anh DD. First report on prevalence and risk factors of severe atypical pneumonia in Vietnamese children aged 1-15 years. BMC Public Health. 2014;14:1304. doi: 10.1186/ s41479-017-0031-4

21. Ishiguro N, Koseki N, Kaiho M, Kikuta H, Togashi T, Oba K, et al. Regional Differences in Prevalence of Macrolide Resistance among Pediatric Mycoplasma pneumoniae Infections in Hokkaido, Japan. Jpn J Infect Dis. 2016;69(3):186-190.

22. Jain S, Williams DJ, Arnold SR, Ampofo K, Bramley AM, Reed $C$, et al. Community-acquired pneumonia requiring hospitalization among U.S. children. N Engl J Med. 2015;372(9):835-845.

23. Jonnalagadda S, Rodríguez O, Estrella B, Sabin LL, Sempértegui $\mathrm{F}$, Hamer $\mathrm{DH}$. Etiology of severe pneumonia in Ecuadorian children. PloS One. 2017;12(2):e0171687.

24. Kicinski P, Wisniewska-Ligier M, Wozniakowska-Gesicka T. Pneumonia caused by Mycoplasma pneumoniae and Chlamydophila pneumoniae in children - comparative analysis of clinical picture. Adv Med Sci. 2011;56(1):56-63.

25. Kogoj R, Mrvic T, Praprotnik M, Kese D. Prevalence, genotyping and macrolide resistance of Mycoplasma pneumoniae among isolates of patients with respiratory tract infections, Central Slovenia, 2006 to 2014. Euro Surveill. 2015;20(37). doi: 10.2807/1560-7917.ES.2015.20.37.30018.

26. Korppi M, Heiskanen-Kosma T, Kleemola M. Incidence of community-acquired pneumonia in children caused by Mycoplasma pneumoniae: serological results of a prospective, population-based study in primary health care. Respirol Carlton Vic. 2004;9(1):109-114.

27. Kurz H, Göpfrich H, Huber K, Krugluger W, Asbott F, Wabnegger $\mathrm{L}$, et al. Spectrum of pathogens of in-patient children and youths with community acquired pneumonia: a 3 year survey of a community hospital in Vienna, Austria. Wien Klin Wochenschr. 2013;125(21-22):674-679.

28. Lassmann B, Poetschke M, Ninteretse B, Issifou S, Winkler $S$, Kremsner PG, et al. Community-acquired pneumonia in children in Lambarene, Gabon. Am J Trop Med Hyg. 2008;79(1):109-114.

29. Liao J-Y, Zhang T. Distribution characteristics of Mycoplasma pneumoniae, Chlamydia pneumoniae, and Legionella pneumophila in hospitalized children with acute respiratory tract infection: an analysis of 13198 cases. Zhongguo Dang Dai Er Ke Za Zhi. 2016;18(7):607-613.

30. Lochindarat S, Suwanjutha S, Prapphal N, Chantarojanasir T, Bunnag T, Deerojanawong J, et al. Mycoplasma pneumoniae and Chlamydophila pneumoniae in children with community-acquired pneumonia in Thailand. Int J Tuberc Lung Dis. 2007;11(7):814-819. 
Merida-Vieyra J et al. M. pneumoniae en pacientes pediátricos

31. Lova R, Naim B, Rakotomahefa M, Andrianirina AZ, Robinson AL, Raobidjaona $\mathrm{H}$, et al. Prevalence of Mycoplasma pneumoniae infection in Malagasy children. Pediatr Infect Dis J. 2017;36(5):467-471.

32. Mathew JL, Singhi S, Ray P, Hagel E, Saghafian-Hedengren S, Bansal A, et al. Etiology of community acquired pneumonia among children in India: prospective, cohort study. J Glob Health. 2015;5(2):050418. doi: 10.7189/jogh.05.020418.

33. Matute AJ, Brouwer WP, Hak E, Delgado E, Alonso E, Hoepelman IM. Aetiology and resistance patterns of community-acquired pneumonia in León, Nicaragua. Int J Antimicrob Agents. 2006;28(5):423-427.

34. Medjo B, Atanaskovic-Markovic M, Radic S, Nikolic D, Lukac M, Djukic S. Mycoplasma pneumoniae as a causative agent of community-acquired pneumonia in children: clinical features and laboratory diagnosis. Ital J Pediatr. 2014;40:104. doi: 10.1186/s13052-014-0104-4

35. Meyer Sauteur PM, Bleisch B, Voit A, Maurer FP, Relly C, Berger C, et al. Survey of macrolide-resistant Mycoplasma pneumoniae in children with community-acquired pneumonia in Switzerland. Swiss Med Wkly. 2014;144:w14041.

36. Sáez-Llorens X, Castaño E, Wubbel L, Castrejón MM, de Morales I, Vallarino D, et al. Importance of Mycoplasma pneumoniae and Chlamydia pneumoniae in children with community-acquired pneumonia. Rev Med Panama. 1998;23(2):27-33.

37. Kenri T, Okazaki N, Yamazaki T, Narita M, Izumikawa K, Matsuoka M, et al. Genotyping analysis of Mycoplasma pneumoniae clinical strains in Japan between 1995 and 2005: type shift phenomenon of M. pneumoniae clinical strains. J Med Microbiol. 2008;57(Pt 4):469-475.

38. Edelstein I, Rachina S, Touati A, Kozlov R, Henin N, Bébéar C, et al. Mycoplasma pneumoniae Monoclonal P1 Type 2c Outbreak, Russia, 2013. Emerg Infect Dis. 2016;22(2):348-350.

39. Meyer Sauteur PM, Unger WWJ, Nadal D, Berger C, Vink C, van Rossum AMC. Infection with and Carriage of Mycoplasma pneumoniae in Children. Front Microbiol. 2016;7:329. doi.org/10.3389/fmicb.2016.00329

40. Diaz MH, Cross KE, Benitez AJ, Hicks LA, Kutty P, Bramley $A M$, et al. Identification of Bacterial and Viral Codetections With Mycoplasma pneumoniae Using the TaqMan Array Card in Patients Hospitalized With Community-Acquired Pneumonia. Open Forum Infect Dis. 2016;3(2):ofw071.

41. Spuesens EBM, Fraaij PLA, Visser EG, Hoogenboezem T, Hop WCJ, van Adrichem LNA, et al. Carriage of Mycoplasma pneumoniae in the upper respiratory tract of symptomatic and asymptomatic children: an observational study. PLoS Med. 2013;10(5):e1001444.

42. Chaudhry R, Ghosh A, Chandolia A. Pathogenesis of Mycoplasma pneumoniae: An update. Indian J Med Microbiol. 2016;34(1):7-16.

43. Jiang W, Qian L, Liang H, Tian M, Liu F, Zhao D. Relationships between the varied ciliated respiratory epithelium abnormalities and severity of Mycoplasma pneumoniae pneumonia. Scand J Infect Dis. 2014;46(7):486-492.

44. Shimizu T. Inflammation-inducing Factors of Mycoplasma pneumoniae. Front Microbiol. 2016;7:414. doi: 10.3389/ fmicb.2016.00414

45. Saraya T. The History of Mycoplasma pneumoniae Pneumonia. Front Microbiol. 2016;7:364. doi: 10.3389/ fmicb.2016.00364

46. Parrott GL, Kinjo T, Fujita J. A Compendium for Mycoplasma pneumoniae. Front Microbiol. 2016;7:513. doi: 10.3389/ fmicb.2016.00513

47. Miyashita N, Narita M, Tanaka T, Akaike H, Teranishi H, Oishi $T$, et al. Histological findings in severe Mycoplasma pneumoniae pneumonia. J Med Microbiol. 2017;66(5):690-692.

48. Dumke R, Jacobs E. Antibody Response to Mycoplasma pneumoniae: Protection of Host and Influence on Outbreaks? Front Microbiol. 2016;7:39. doi: 10.3389/ fmicb.2016.00039

49. Simecka J. What we have Learned from Animal Models of Mycoplasma pneumoniae Disease: Virulence Mechanisms and Host Responses. Curr Pediatr Rev. 2013;9(4):314-323.

50. Saraya T, Kurai D, Nakagaki K, Sasaki Y, Niwa S, Tsukagoshi $\mathrm{H}$, et al. Novel aspects on the pathogenesis of Mycoplasma pneumoniae pneumonia and therapeutic implications. Front Microbiol. 2014;5:410.

51. Wang $M$, Wang $Y$, Yan $Y$, Zhu C, Huang L, Shao X, et al. Clinical and laboratory profiles of refractory Mycoplasma pneumoniae pneumonia in children. Int J Infect Dis. 2014;29:18-23.

52. Waites KB, Atkinson TP. The role of Mycoplasma in upper respiratory infections. Curr Infect Dis Rep. 2009;11(3):198206.

53. Narita M. Classification of Extrapulmonary Manifestations Due to Mycoplasma pneumoniae Infection on the Basis of Possible Pathogenesis. Front Microbiol. 2016;7:23.

54. Esposito S, Cavagna R, Bosis S, Droghetti R, Faelli N, Principi $\mathrm{N}$. Emerging role of Mycoplasma pneumoniae in children with acute pharyngitis. Eur J Clin Microbiol Infect Dis. 2002;21(8):607-610.

55. Kishaba T. Community-Acquired Pneumonia Caused by Mycoplasma pneumoniae: How Physical and Radiological Examination Contribute to Successful Diagnosis. Front Med. 2016;3:28.

56. Wang K, Gill P, Perera R, Thomson A, Mant D, Harnden A. Clinical symptoms and signs for the diagnosis of Mycoplasma pneumoniae in children and adolescents with community-acquired pneumonia. Cochrane Database Syst Rev. 2012;10:CD009175.

57. Yeh J-J, Wang Y-C, Hsu W-H, Kao C-H. Incident asthma and Mycoplasma pneumoniae: A nationwide cohort study. J Allergy Clin Immunol. 2016;137(4):1017-1023.e1-6. 
58. Izumikawa K. Clinical Features of Severe or Fatal Mycoplasma pneumoniae Pneumonia. Front Microbiol. 2016;7:800.

59. Romero Reyes LE. Neumonía por Mycoplasma pneumoniae y Chlamydophila pneumoniae [Subpespecialidad en Infectología Pediátrica]. [Ciudad de México]: Universidad Nacional Autónoma de México; 2016.

60. Chiu C-Y, Chen C-J, Wong K-S, Tsai M-H, Chiu C-H, Huang Y-C. Impact of bacterial and viral coinfection on mycoplasmal pneumonia in childhood community-acquired pneumonia. J Microbiol Immunol Infect. 2015;48(1):51-56.

61. Gong L, Zhang C-L, Zhen Q. Analysis of clinical value of CT in the diagnosis of pediatric pneumonia and mycoplasma pneumonia. Exp Ther Med. 2016;11(4):1271-1274.

62. Lu A, Wang C, Zhang X, Wang L, Qian L. Lactate Dehydrogenase as a Biomarker for Prediction of Refractory Mycoplasma pneumoniae Pneumonia in Children. Respir Care. 2015;60(10):1469-1475.

63. Vervloet LA, Marguet C, Camargos PAM. Infection by Mycoplasma pneumoniae and its importance as an etiological agent in childhood community-acquired pneumonias. Braz J Infect Dis. 2007;11(5):507-514.

64. Atkinson TP, Balish MF, Waites KB. Epidemiology, clinical manifestations, pathogenesis and laboratory detection of Mycoplasma pneumoniae infections. FEMS Microbiol Rev. 2008;32(6):956-973.

65. Loens K, Goossens H, leven M. Acute respiratory infection due to Mycoplasma pneumoniae: current status of diagnostic methods. Eur J Clin Microbiol Infect Dis. 2010;29(9):1055-1069.

66. Waites KB, Balish MF, Atkinson TP. New insights into the pathogenesis and detection of Mycoplasma pneumoniae infections. Future Microbiol. 2008;3(6):635-648.

67. Daxboeck F, Stadler M, Assadian O, Marko E, Hirschl AM, Koller W. Characterization of clinically isolated Ralstonia mannitolilytica strains using random amplification of polymorphic DNA (RAPD) typing and antimicrobial sensitivity, and comparison of the classification efficacy of phenotypic and genotypic assays. J Med Microbiol. 2005;54(Pt 1):55-61.

68. Diaz MH, Winchell JM. The Evolution of Advanced Molecular Diagnostics for the Detection and Characterization of Mycoplasma pneumoniae. Front Microbiol. 2016;7:232.
69. Pereyre S, Goret J, Bébéar C. Mycoplasma pneumoniae: Current Knowledge on Macrolide Resistance and Treatment. Front Microbiol. 2016;7:974.

70. Spuesens EBM, Meyer Sauteur PM, Vink C, van Rossum AMC. Mycoplasma pneumoniae infections--does treatment help? J Infect. 2014;69 (1):S42-46.

71. Biondi E, McCulloh R, Alverson B, Klein A, Dixon A, Ralston $S$. Treatment of mycoplasma pneumonia: a systematic review. Pediatrics. 2014;133(6):1081-1090.

72. Gardiner SJ, Gavranich JB, Chang AB. Antibiotics for community-acquired lower respiratory tract infections secondary to Mycoplasma pneumoniae in children. Cochrane Database Syst Rev. 2015;1:CD004875.

73. Matsubara K, Morozumi M, Okada T, Matsushima T, Komiyama O, Shoji M, et al. A comparative clinical study of macrolide-sensitive and macrolide-resistant Mycoplasma pneumoniae infections in pediatric patients. J Infect Chemother. 2009;15(6):380-383.

74. Zhou Y, Zhang Y, Sheng Y, Zhang L, Shen Z, Chen Z. More complications occur in macrolide-resistant than in macrolide-sensitive Mycoplasma pneumoniae pneumonia. Antimicrob Agents Chemother. 2014;58(2):1034-1038.

75. Citti C, Blanchard A. Mycoplasmas and their host: emerging and re-emerging minimal pathogens. Trends Microbiol. 2013;21(4):196-203.

76. Okazaki N, Narita M, Yamada S, Izumikawa K, Umetsu M, Kenri T, et al. Characteristics of macrolide-resistant Mycoplasma pneumoniae strains isolated from patients and induced with erythromycin in vitro. Microbiol Immunol. 2001;45(8):617-620.

77. Ishiguro N, Koseki N, Kaiho M, Ariga T, Kikuta H, Togashi T, et al. Therapeutic efficacy of azithromycin, clarithromycin, minocycline and tosufloxacin against macrolide-resistant and macrolide-sensitive Mycoplasma pneumoniae pneumonia in pediatric patients. PloS One. 2017;12(3):e0173635.

78. Jensen KE, Senterfit LB, Chanock RM, Smith CB, Purcell RH. An inactivated Mycoplasma pneumoniae vaccine. JAMA. 1965;194(3):248-252.

79. Hausner M, Schamberger A, Naumann W, Jacobs E, Dumke R. Development of protective anti-Mycoplasma pneumoniae antibodies after immunization of guinea pigs with the combination of a P1-P30 chimeric recombinant protein and chitosan. Microb Pathog. 2013;64:23-32. 